\title{
Periostin regenerates broken heart
}

DOI:

10.1038/nrd2406
Myocardial infarction, which results in scar formation rather than regeneration of the lost cardiomyocytes responsible for the 'pump' function of the heart, is a major cause of heart failure. Kühn and colleagues, writing in Nature Medicine, now show that following myocardial infarction, cardiomyocytes can be induced to proliferate by a recombinant protein known as periostin, resulting in improved cardiac function. Periostin and the pathway it induces could thus represent a novel therapeutic target for heart failure.

Following observations that cardiomyocytes in the border zone of a myocardial infarction have transiently increased cell-cycle activity, the authors hypothesized that

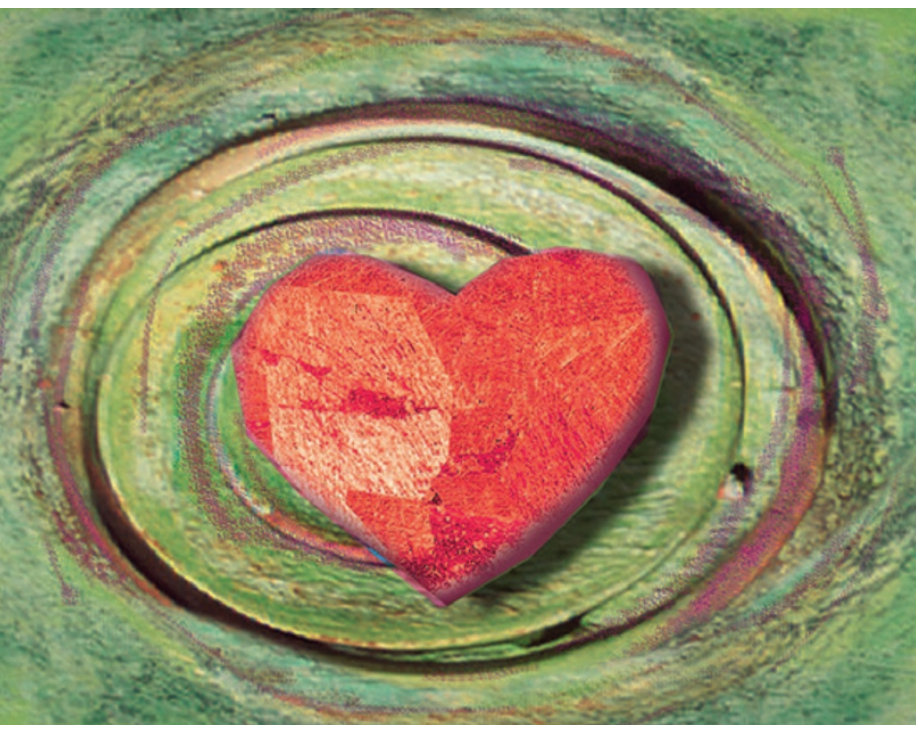

it may be possible to apply extracellular factors to induce cardiomyocyte proliferation. The authors identified periostin - a protein normally expressed during cardiac development but also re-expressed following injury to adult tissue including the myocardium - as a potential factor for inducing proliferation.

In vitro studies showed that periostin induces the full mitotic cell cycle of differentiated mononucleated rat cardiomyocytes. Further examinations showed that periostin requires integrin $\alpha_{v}$ and a $\beta_{1}, \beta_{3}$ or $\beta_{5}$ subunit to induce cell-cycle re-entry via the phosphatidylinositol-3 kinase (PI3K) pathway. Inhibition of AKT signalling, an important downstream target of PI3K, also reduced periostin-induced cell-cycle re-entry. To confirm these results, adenoviral transduction of the bifunctional phosphatase PTEN, known to regulate the PI3K pathway, abolished periostin-stimulated DNA synthesis in cardiomyocytes. Also, transduction of a constitutively active form of PI3K increased cardiomyocyte DNA synthesis in the absence of periostin, indicating that PI3K signalling is sufficient for cell-cycle re-entry in the absence of periostin.

To determine whether periostin stimulates cardiomyocyte cell-cycle re-entry in vivo, recombinant periostin was injected into the myocardium of rats, resulting in cycling mononucleated cardiomyocytes with periostin-induced DNA synthesis and cytokinesis near the injection site.
As it had been previously suggested that sustained cardiomyocyte cellcycle activity may decrease infarct size following myocardial infarction, Kühn and colleagues developed a long-term delivery system in which periostin is associated with Gelfoam, a biodegradable extracellular matrix.

In a rat model of myocardial infarction, periostin bound to Gelfoam was administered epicardially at the time of heart injury. Twelve weeks later, the periostin-treated rats had improved ventricular remodelling, better myocardial function and reduced infarct size. In periostintreated hearts, these improvements were attributed to the 100 -fold higher proportion of cycling cardiomyocytes compared with apoptotic cardiomyocytes 12 weeks after the myocardial infarction. Also, compared with the control hearts, the periostin-treated hearts had $\sim 6$ million more cardiomyocyte nuclei. Based on this, the authors concluded that cell-cycle re-entry and division of differentiated cardiomyocytes can account for the periostin-induced functional and structural improvements, which may provide an approach to induce myocardial repair.

Bethan Hughes

ORIGINAL RESEARCH PAPER Kühn, B. et al. Periostin induces proliferation of differentiated cardiomyocytes and promotes cardiac repair. Nature Med. 13, 962-969 (2007) FURTHER READING Kaye, D. M. et al. Drug discovery for heart failure: a new era or the end of the pipeline? Nature Rev. Drug Discov. 6, 127-139 (2007) 